\title{
Characterization of a QTL affecting skeletal size in mice
}

\author{
Julian K. Christians, Victoria Bingham, Fiona Oliver, Thomas T. Heath, Peter D. Keightley
}

Institute of Cell, Animal and Population Biology, University of Edinburgh, Ashworth Laboratories, King's Buildings, West Mains Road, Edinburgh, EH9 3JT, UK

Received: 9 July 2002 / Accepted: 12 November 2002

\begin{abstract}
Previous work identified a tail length QTL on Chromosome (Chr) 1 in an $\mathrm{F}_{2}$ population of C57BL/ $6 \mathrm{~J} \times \mathrm{DBA} / 2 \mathrm{~J}$ mice. The goals of the present study were to (1) refine the position of this QTL by additional genotyping of samples from the original study; (2) confirm the effect of this QTL by producing a partially congenic strain carrying the C57BL/6J allele against the DBA/2J background; and (3) examine the effect of the QTL on skeletal dimensions. The presence of the QTL was confirmed in a new $F_{2}$ population $(\mathrm{N}=431)$ derived from the partially congenic strain, and estimates of its additive effects were similar to those from the original $F_{2}$ population $(\mathrm{N}=901)$ in both sexes, i.e., the C57BL/6J chromosomal segment increased tail length, the additive effect (half the difference between homozygotes) being $0.5-0.8$ standard deviations. The QTL region was more than halved, relative to that in the previous study, to an 8-cM region between D1Mit30 and D1Mit57. Among a subsample of individuals $(\mathrm{N}=30)$ from the new $\mathrm{F}_{2}$ population that were not recombinant within the QTL region, there was a significant additive effect of the QTL on the length of the humerus, femur, tibia, mandible, scapula, pelvic girdle, and a tail bone; the direction of the effect was the same as for tail length. No significant effect was found on the number of bones in the tail or on the dimensions of the ulna, skull, or first vertebra.
\end{abstract}

\section{Introduction}

Many traits of medical, agricultural or evolutionary significance show continuous variation (e.g., body size), rather than having discrete, one-or-theother states (e.g., blood types). In recent years, numerous studies have used molecular markers to

Correspondence to: J. K. Christians; E-mail: julian.christians@ ed.ac.uk identify chromosomal regions that influence continuous variation in a variety of traits (i.e., quantitative trait loci, or QTL) (Mackay 2001). A large number of QTL studies of mammalian species have focused on body mass (Corva and Medrano 2001) or adiposity (Barsh et al. 2000), and yet relatively little work has examined a related but distinct class of trait: structural size (e.g., bone length) (Corva and Medrano 2001; but see Cheverud et al. 2001; Drake et al. 2001; Klingenberg et al. 2001). Morris et al. (1999) examined tail length in an $F_{2}$ cross between two inbred strains of mice (C57BL/6J and DBA/2J) and detected a QTL on Chr 1 with a particularly large effect on this trait (1.3 phenotypic standard deviations between homozygotes), which accounts for $21 \%$ of the phenotypic variation. The QTL also has an effect on body mass at 10 weeks of age that is somewhat smaller than the effect on tail length (Morris et al. 1999).

The magnitude of the effect reported by Morris et al. (1999) is unusually large in comparison with many QTL studies of growth and mass in mice (see Corva and Medrano 2001), and this would be expected to facilitate the identification of the underlying genes. However, at least two caveats must be made regarding this result. Firstly, this QTL was mapped by selective genotyping, i.e., genotyping of only the most phenotypically extreme individuals (Darvasi 1997). While this approach improves the efficiency with which QTL may be detected, it reduces the power to detect multiple QTL within a chromosomal region; individuals that are recombinant between two QTL with effects in the same direction tend to have intermediate phenotypes and so are not genotyped, reducing the power to resolve the two QTL. Secondly, even if the observed effect is due to a single QTL, there is a general tendency for estimates of QTL effects to be biased upwards because the same data are used to identify whether a QTL is present and to estimate its phenotypic effect (Mackay 2001). Replication is 
therefore important in order to confirm the magnitude of the phenotypic effect.

In light of the above, the goals of the present study were to (1) refine the position of the tail length QTL by additional genotyping of samples from the original study (Morris et al. 1999), thereby increasing the power to detect multiple QTL; (2) confirm the effect of this QTL by producing a partially congenic strain containing the $\mathrm{C} 57 \mathrm{BL} / 6 \mathrm{~J}$ allele of the QTL against the DBA/2J background and then producing a new $\mathrm{F}_{2}$ population derived from this partially congenic strain; and (3) further characterize the effect of the QTL by examining its effect on various skeletal dimensions. To examine whether the QTL affected only the tail or had more general effects on the skeleton, three groups of traits were examined: (A) traits associated with the tail/spine, i.e., the dimensions of a vertebra and a tail bone and the number of bones in the tail; (B) the dimensions of long bones (sensu Sims and Baron 2000), i.e., the humerus, ulna, femur, and tibia; and $(C)$ the dimensions of flat bones (sensu Sims and Baron 2000), i.e., the skull, mandible, scapula, and pelvic girdle.

\section{Materials and methods}

Original $\boldsymbol{F}_{2}$ population. General animal maintenance was as described by Bünger et al. (2001). The original $\mathrm{F}_{2}$ population was derived from the inbred strains C57BL/6J (C57) and DBA/2J (DBA) and is described in more detail by Morris et al. (1999). Body mass was recorded at 3,6 , and 10 weeks, and tail length was measured at 10 weeks in 901 individuals from first and second parity litters of 67 families. The sample size described here is slightly smaller than that in the original study (927) because of missing samples.

Production of partially congenic strain and new $\boldsymbol{F}_{2}$ population. A partially congenic strain was produced by backcrossing to DBA for four generations while selecting for the C57 allele at markers at each end of the target region, as identified in the original study (Morris et al. 1999), i.e., D1Mit7 and D1Mit57, and selecting against the C57 allele at markers flanking the region, i.e., D1Mit213 and D1Mit150. Markers at approximately 10-cM intervals between D1Mit7 and D1Mit57 were also genotyped (D1Mit80, D1Mit191, D1Mit30, and D1Mit42) to ensure that the entire region was introgressed. After four generations of backcrossing, the contribution from the C57 autosomal genome was expected to be about $6.3 \%$. The fourth backcross generation was intercrossed to obtain animals that were homozygous C57 for the introgressed region, and the par- tially congenic strain was then maintained for 12 generations. This strain was then crossed to DBA, and the resulting $F_{1}$ population was intercrossed to produce a new $F_{2}$ population, in which tail length and body mass were measured at 3, 6, and 10 weeks in 431 individuals from 30 families (including first through sixth parity litters).

Microsatellite genotyping. Extraction of genomic DNA from either ear clip or spleen tissue and amplification of microsatellite markers were performed by standard methods. PCR products were separated on 20-cm polyacrylamide gels, stained with ethidium bromide, and photographed under UV light. Photographs were scored twice, and ambiguous genotypes were re-amplified.

Markers genotyped. In the original study (Morris et al. 1999), the entire $F_{2}$ population was genotyped at D1Mit30. In addition, the most extreme individuals (the highest and lowest 50 individuals on the basis of 10 -week weight and fat percentage within each sex) were genotyped at five other markers on Chr 1: D1Mit118, D1Mit122, D1Mit7, D1Mit57, and D1Mit155 (see Table 1 for marker locations). In the present study, the remaining individuals in this population were genotyped at D1Mit7 and D1Mit57, and all individuals were genotyped at four additional markers: D1Mit48, D1Mit157, D1Mit42, and D1Mit150 (see Table 1). Preliminary analyses indicated that the QTL was in the region between D1Mit30 and D1Mit57, and therefore individuals that were recombinant between D1Mit30 and D1Mit42 were genotyped at D1Mit265, while individuals recombinant between D1Mit42 and D1Mit57 were genotyped at D1Mit14 and D1Mit106 (Table 1).

All individuals from the new $\mathrm{F}_{2}$ population were genotyped at D1Mit7, D1Mit30, D1Mit42, and D1Mit57. As with individuals from the original $\mathrm{F}_{2}$ population, recombinant individuals were genotyped at D1Mit265 or D1Mit14 and D1Mit106, depending on the location of recombination.

Linkage map. The order of the markers used in this study was obtained from the Mouse Genome Database (MGD 2002) and the Ensembl Mouse Genome Server Database (Ensembl 2002). This order was assumed to be correct, since the genetic and physical maps are in agreement, except that D1Mit118 is not mapped on the physical map. Map positions for these markers were calculated with the software package CRIMAP (Green et al. 1990), combining genotypes from the original and new $\mathrm{F}_{2}$ populations, and are shown in Table 1. 
Table 1. Microsatellite markers genotyped in the original and new $F_{2}$ populations

\begin{tabular}{llll}
\hline & & \multicolumn{2}{c}{ Samples genotyped } \\
\cline { 3 - 4 } Marker & Map position $(\mathrm{cM})^{a}$ & Original F $_{2}$ population & New $F_{2}$ population \\
\hline D1Mit118 & 0.0 & Selected $^{\mathrm{b}}$ & None \\
D1Mit122 & 14.7 & Selected & None \\
D1Mit7 & 28.3 & All & All \\
D1Mit48 & 33.9 & All & None \\
D1Mit157 & 37.2 & All & None \\
D1Mit30 & 45.0 & All & All \\
D1Mit265 & 50.4 & Recombinants & Recombinants \\
D1Mit42 & 53.3 & All & All \\
D1Mit14 & 54.8 & Recombinants $^{\mathrm{c}}$ & Recombinants $^{\mathrm{c}}$ \\
D1Mit106 & 56.2 & Recombinants $^{\mathrm{c}}$ & Recombinants $^{\mathrm{c}}$ \\
D1Mit57 & 58.0 & All & All \\
D1Mit150 & 67.9 & All & All \\
D1Mit155 & 89.3 & Selected & None $^{\mathrm{b}}$ \\
\hline
\end{tabular}

${ }^{a}$ Calculated by using CRIMAP (Green et al. 1990) combining genotypes from the original and new $\mathrm{F}_{2}$ populations.

b Based on 10-week body weight and fat percentage as described by Morris et al. (1999).

${ }^{c}$ Only recombinants between adjacent markers were genotyped.

d Samples were genotyped but not included in interval mapping because not all $\mathrm{F}_{1}$ parents were heterozygous (see text).

Interval mapping. Interval mapping (one- and two-QTL analysis) was performed by using the QTL Express package (Seaton et al. 2002). The two-QTL analysis of QTL Express estimates the effects of two QTL at separate positions simultaneously, examining all possible pairs of locations, and determines the pair of locations for which the model explains the most variation; this analysis provides F-statistics for the tests of two QTL versus no QTL and of two QTL versus one QTL; the two-QTL analyses in this study used a 1-cM grid. Composite interval mapping was used as an alternative method to search for multiple QTL and was performed with QTL Cartographer v. 1.21 (Wang et al. 2001). In analyses of the original $F_{2}$ population with QTL Express, QTL effects and the effects of parental pair, parity, and a linear covariate for litter size at weaning were fitted simultaneously. Composite interval mapping was performed on the residuals of tail length from a general linear model including pair, parity, and litter size (calculated by using proc GLM, SAS Institute, 1989), with three background control markers and a window size of 5 $\mathrm{cM}$. In the analysis of the new $\mathrm{F}_{2}$ population, the number of individuals in the post-weaning cage was included as a linear covariate in addition to the factors described above (except in analyses of 3-week trait values). Chromosome-wide significance thresholds for each trait were determined empirically by permuting the marker data, as described by Churchill and Doerge (1994); the threshold was obtained from 500 permutations. The significance of a second QTL in interval mapping was assessed by using the threshold F-value determined for a single-QTL analysis. Threshold F-values are presented as $\mathrm{F}_{0.05}$ and $\mathrm{F}_{0.01}$ for significance at $\alpha=0.05$ and $\alpha=0.01$, respec- tively. Confidence intervals for the location of QTL from interval mapping were calculated from 500 bootstrapped samples (Visscher et al. 1996) and were obtained by using QTL Express.

Effects of QTL on skeletal dimensions. Interval mapping of the original population refined the QTL to the region between D1Mit30 and D1Mit57 (see below), and, therefore, individuals that were not recombinant within this region, i.e., those with the same genotype at D1Mit30, D1Mit42, and D1Mit57, were killed at 11 weeks of age and immediately frozen. Ten first and second litters that included non-recombinant individuals of each of the three genotypes (DBA/DBA, DBA/C57, C57/C57) were selected, and from each of these litters, one individual of each genotype was selected at random for analysis of skeletal dimensions; each of the 10 litters was from different pairs of $F_{1}$ parents. Owing to small litter sizes, it was not possible to select individuals from only one sex; 11 females and 19 males were selected. Pooling the sexes was justified, given the similar estimates of the location and effect of tail-length QTL in both sexes (see below).

Selected individuals were thawed, and the length of the tail was measured. The carcasses were skinned and placed in a water bath at $80^{\circ} \mathrm{C}$ overnight. The soft tissue was stripped from the skeleton with forceps, and the following skeletal dimensions were measured with calipers $( \pm 0.02 \mathrm{~mm})$ : the length (along the anterior-posterior axis) and width of the first cervical vertebra (i.e., atlas), and the lengths of the humerus, ulna, femur, tibia, scapula (from the tip of the acromion process to the edge of the intraspinous fossa) and pelvic girdle (from the tip of the 
Table 2. Means and standard deviations for tail length and body mass at various ages in the original and new $F_{2}$ populations

\begin{tabular}{|c|c|c|c|c|c|c|}
\hline & \multicolumn{3}{|c|}{ Females } & \multicolumn{3}{|c|}{ Males } \\
\hline & $N$ & Mean & $\begin{array}{l}\text { Standard } \\
\text { deviation }\end{array}$ & $N$ & Mean & $\begin{array}{l}\text { Standard } \\
\text { deviation }\end{array}$ \\
\hline \multicolumn{7}{|l|}{ Original $F_{2}$ population } \\
\hline 10-week tail length $(\mathrm{mm})$ & 454 & 72.84 & 3.77 & 447 & 75.83 & 3.93 \\
\hline 10 -week body mass $(\mathrm{g})$ & 454 & 22.24 & 1.98 & 447 & 29.33 & 2.81 \\
\hline \multicolumn{7}{|l|}{ New $F_{2}$ population } \\
\hline 10-week tail length $(\mathrm{mm})$ & 162 & 79.73 & 3.29 & 187 & 81.27 & 3.57 \\
\hline 6-week tail length ( $\mathrm{mm})$ & 169 & 72.98 & 4.17 & 180 & 73.71 & 4.68 \\
\hline 3-week tail length (mm) & 167 & 57.24 & 5.08 & 195 & 56.22 & 5.84 \\
\hline 10-week body mass (g) & 162 & 21.79 & 1.87 & 187 & 26.94 & 2.72 \\
\hline 6-week body mass (g) & 169 & 18.12 & 1.81 & 180 & 21.59 & 2.99 \\
\hline 3-week body mass (g) & 167 & 9.95 & 2.01 & 195 & 9.53 & 2.36 \\
\hline
\end{tabular}

ischium to the tip of the ilium). Where possible, the bones from the left side of the body were used. Skull length (from the tip of the nasal bones to the tip of the frontal bones), width (the width of the frontal bones), and mandible length (between landmarks 2 and 5 in Klingenberg et al. 2001) were also measured. The lower spinal column and tail were digested in a proteinase $\mathrm{K}$ solution, and the number of bones from the beginning of the sacrum to the tip of the tail was counted with the aid of a dissecting microscope. The length of the tenth caudal bone of the tail /counting from the first bone of the sacrum) was also measured. All measurements were made in triplicate.

Linear measurements were analyzed by using a general linear model (proc GLM, SAS Institute, 1989) that included the effects of genotype, parental pair, and sex. The additive effects (i.e., half the difference between the homozygotes) and dominance effects (i.e., the difference between the heterozygote and the mean of the homozygotes) were estimated by using the "estimate" statement of proc GLM (SAS Institute 1989), which also calculated the P-value under the null hypothesis that the true effect size was zero. To test for differences between genotypes in the number of bones from the sacrum to the tip of the tail, a Kruskal-Wallis test (proc NPAR1WAY, SAS Institute 1989/ was employed. Because of the exploratory nature of this part of the study, it was desirable to examine the effect of genotype on each individual character, and therefore multivariate analyses were not performed. A significance level of $\alpha=0.05$ was used for each test, and therefore we accept that, on examination of 14 traits, the expectation is that about one trait will be significant at the $5 \%$ level owing to chance.

\section{Results}

QTL locations and effects-original $F_{2}$ population. The phenotypic means and standard deviations

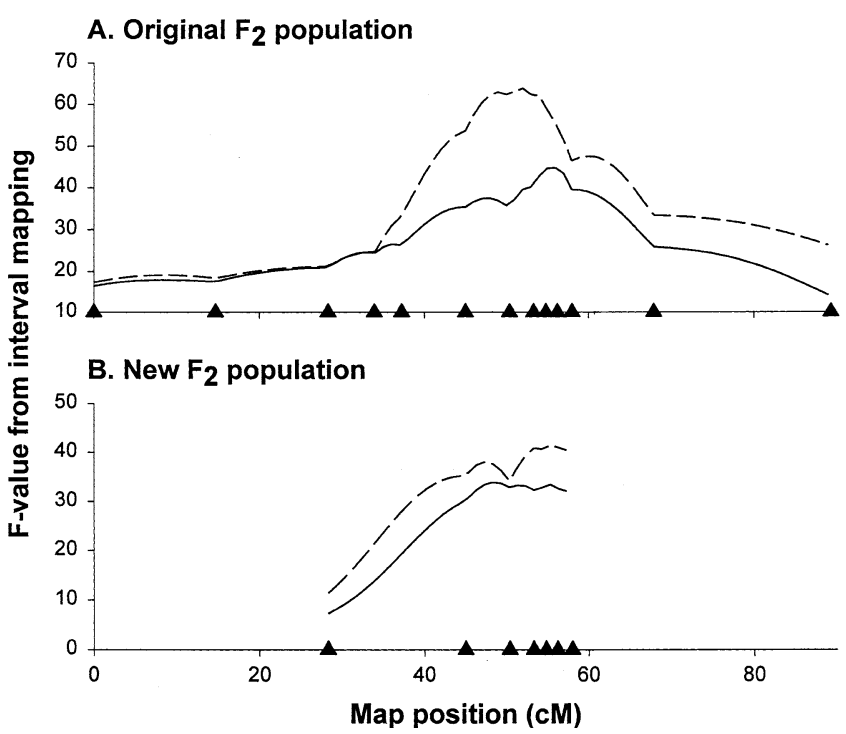

Fig. 1. F-value and LOD score plots for 10 -week tail length from interval mapping of the original (panel A) and new $\mathrm{F}_{2}$ populations (panel B) for females (solid lines) and males (dashed lines). Triangles denote the positions of markers typed in each experiment. Thresholds for the chromosome-wide significance level are not shown because the entire plots exceeded the threshold value for $\alpha=0.01$.

for the measured growth traits are shown in Table 2. For both females and males, the peak F value $(44.8$ and 63.8 , respectively) from single-QTL analyses of 10 -week tail length greatly exceeded the threshold of significance at $\alpha=0.01$ determined by permutation analysis $\left(\mathrm{F}_{0.01}=6.5\right.$ for both sexes $)$; i.e., there was substantial support for the presence of at least one QTL (Fig. 1A). In the two-QTL analysis, the F-value for the addition of a second QTL was not significant for females $\left(F=3.7\right.$ vs. $\left.F_{0.05}=4.9\right)$ or males $(F=4.1$ vs. $\left.F_{0.05}=4.5\right)$. Similarly, composite interval mapping did not reveal more than one significant peak for either sex (data not shown).

In both sexes, the C57 homozygotes had longer tails than the DBA homozygotes, although the esti- 
mate of the additive effect was slightly higher in males (Table 3). The dominance effect was relatively close to zero in males, whereas in females the C57 allele was partially dominant (Table 3). Although the estimates of the position of the QTL differed slightly between the sexes (Table 3), the bootstrapping distributions overlapped substantially $195 \%$ confidence interval for females: $43 \mathrm{cM}$ to $58 \mathrm{cM}$; for males: $47 \mathrm{cM}$ to $55 \mathrm{cM}$ ).

Given that the estimates and locations of the QTL were similar in both sexes, the data were pooled and reanalyzed together (including sex as a term in the analysis). Single-QTL analysis of 10 -week tail length was highly significant $(\mathrm{F}=108.3$ vs. $\left.\mathrm{F}_{0.01}=6.6\right)$ and yielded results similar to the sexspecific analyses (location: $54 \mathrm{cM}$; 95\% confidence interval: $48 \mathrm{cM}$ to $56 \mathrm{cM}$; additive effect: $-0.55 \pm$ 0.04 standard deviation units; dominance effect: $0.21 \pm 0.05$ standard deviation units). In the twoQTL analysis, the F-statistic for the test of two QTL versus one QTL was significant $(\mathrm{F}=5.2$ vs. $\mathrm{F}_{0.05}=4.91$. One QTL was similar to that identified in the single QTL analysis (location: $56 \mathrm{cM}$; additive effect: $-0.48 \pm 0.04$ standard deviation units; dominance effect: $0.22 \pm 0.05$ standard deviation units), whereas the second QTL had a smaller effect (location: $1 \mathrm{cM}$; additive effect: $-0.22 \pm 0.06$ standard deviation units; dominance effect: $0.01 \pm 0.11$ standard deviation units); the region of high support for the smaller QTL was relatively broad compared with that for the larger QTL (data not shown). In contrast to the two-QTL analysis, composite interval mapping of the 10-week tail length data from both sexes combined revealed a single significant peak (data not shown).

Analysis of 10-week body mass revealed significant QTL in females and males, although the support was much weaker than for tail length (females: $\mathrm{F}=8.8$ vs. $\mathrm{F}_{0.01}=6.3$; males: $\mathrm{F}=12.7$ vs. $\mathrm{F}_{0.01}=6.3$; Fig. 2), reflecting smaller effect sizes (Table 3). The additive effects were in the same direction as tail length and were similar in both sexes. In contrast to the tail length results, the estimates of the dominance effects were relatively close to zero in both sexes. The 95\% confidence intervals for the location of the 10-week body mass QTL in females and males overlapped substantially (females: $15 \mathrm{cM}$ to $56 \mathrm{cM}$; males: $0 \mathrm{cM}$ to $58 \mathrm{cM})$. Combining the sexes, the 10 week body mass QTL was highly significant $\left(\mathrm{F}=19.7\right.$ vs. $\left.\mathrm{F}_{0.01}=7.2\right)$, but its magnitude was still relatively small (location: $48 \mathrm{cM}$; additive effect: $-0.15 \pm 0.03$ standard deviation units; dominance effect: $0.09 \pm 0.04$ standard deviation units). The estimated location for the mass QTL was within the $95 \%$ confidence interval for 10-week tail length (see

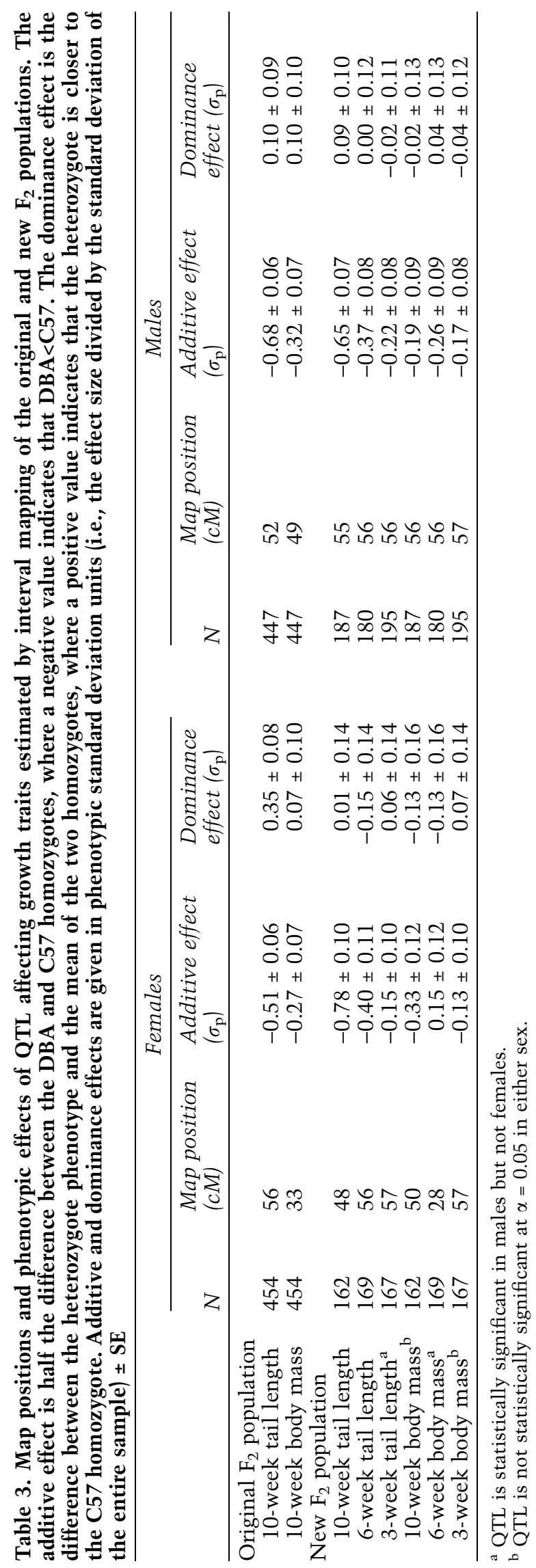




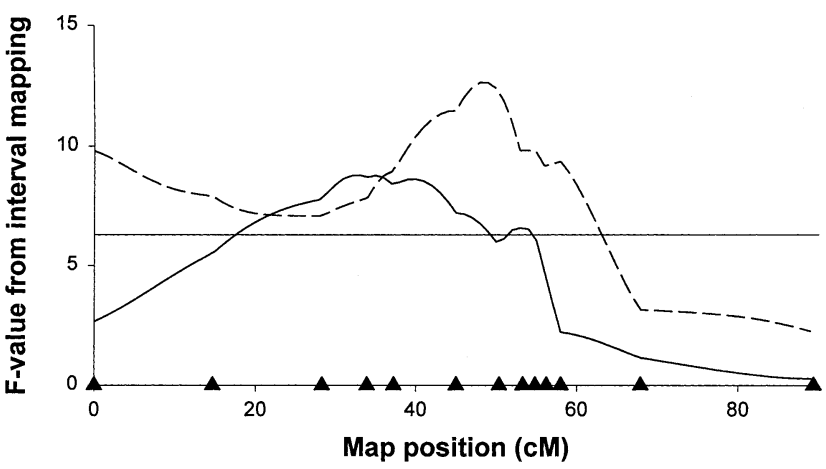

Fig. 2. F-value plot for 10 -week body mass from interval mapping for females (solid line) and males (dashed line) of the original $\mathrm{F}_{2}$ population. Triangles denote the positions of markers typed. The horizontal line indicates the chromosome-wide $1 \%$ significance level obtained by permutation analysis (same for females and males).

above); thus, it is not clear whether the observed effects on tail length and body mass are due to separate QTL or to the pleiotropic effects of a single gene.

Partially congenic strain and $F_{1}$ population. Interval mapping of the new $\mathrm{F}_{2}$ population was based on the assumption that the $F_{1}$ population (produced by crossing the partially congenic strain to DBA) was heterozygous throughout the region examined. For markers within the target region, all three genotypes (DBA/DBA, DBA/C57, C57/C57) were found within most $\mathrm{F}_{2}$ families; where this was not the case, both $F_{1}$ parents were genotyped, and in all of these cases both parents were found to be heterozygous. At the proximal flanking marker, D1Mit213 all $\mathrm{F}_{1}$ individuals genotyped were homozygous for the DBA allele. However, at the distal flanking marker, D1Mit150, some but not all of the $F_{1}$ population were heterozygous. In order that twomarker analyses could be performed with D1Mit150 (see below), the entire $F_{2}$ population was typed at this marker, except where both $\mathrm{F}_{1}$ parents were homozygous.

\section{QTL locations and effects-new $F_{2}$ popula-} tion. As in the original $\mathrm{F}_{2}$ population, there was strong support for a QTL affecting 10-week tail length in both females $\left(\mathrm{F}=33.9\right.$ vs. $\left.\mathrm{F}_{0.01}=5.3\right)$ and males $\left(F=41.3\right.$ vs. $F_{0.01}=5.7$; Fig. $\left.1 B\right)$ in the new $F_{2}$ population produced by crossing the partially congenic strain to DBA; i.e., the introgression of the QTL was successful. The F value for the addition of a second QTL was not significant for either sex (females: $\mathrm{F}=2.4$ vs. $\mathrm{F}_{0.05}=3.9$; males: $\mathrm{F}=2.5$ vs. $\left.\mathrm{F}_{0.05}=3.8\right)$ and, similarly, composite interval mapping did not reveal more than one significant peak (data not shown).
The estimates of the additive effects were similar in sign and in magnitude to those from the original experiment, although the additive effect was slightly higher in females (Table 3). However, the estimates of the dominance effects were relatively close to zero in both sexes (Table 3). The 95\% confidence intervals for the locations of the QTL in the new $\mathrm{F}_{2}$ population (females: $45 \mathrm{cM}$ to $57 \mathrm{cM}$; males: $43 \mathrm{cM}$ to $57 \mathrm{cM}$ ) were similar to those in the original $\mathrm{F}_{2}$ population (see above). It should be noted that this analysis did not include any markers distal to D1Mit57 (see above). To delimit this end of the QTL, a two-marker analysis was performed by including genotypes at D1Mit57 and D1Mit150 in a general linear model (parental pair, parity, litter size at weaning and number of mice in rearing cage were also included in the model). There was a highly significant effect of genotype at D1Mit57 but not at D1Mit150 within both sexes (data not shown). Thus, while it is possible that the QTL lies outside D1Mit57, it is more closely linked to D1Mit57 than to D1Mit150.

There was significant support for a QTL affecting 6-week tail length in both sexes (females: $F=7.0$ vs. $\mathrm{F}_{0.01}=5.9 ; \quad$ males: $\mathrm{F}=11.9$ vs. $\left.\quad \mathrm{F}_{0.01}=5.8\right)$. For 3 -week tail length, the peak F-value just reached the threshold for significance at $\alpha=0.05$ for males $\left(\mathrm{F}=4.0\right.$ vs. $\left.\mathrm{F}_{0.05}=4.0\right)$, but not for females $(\mathrm{F}=1.3$ vs. $\left.\mathrm{F}_{0.05}=4.1\right)$. The estimated additive effects are similar in both sexes and increase with age (Table 3). The estimates for the locations of the tail length QTL at various ages are generally very similar and in all cases are within the $95 \%$ confidence interval for the 10-week QTL (Table 3; see above).

There were no significant QTL for body mass at 10 weeks (females: $\mathrm{F}=3.7$ vs. $\mathrm{F}_{0.05}=4.3$; males: $\mathrm{F}=2.2$ vs. $\mathrm{F}_{0.05}=4.6$ ) or at 3 weeks (females: $\mathrm{F}=1.0$ vs. $\mathrm{F}_{0.05}=4.2$; males: $\mathrm{F}=2.1$ vs. $\left.\mathrm{F}_{0.05}=4.3\right)$. There was a significant QTL for 6-week body mass in males $\left(F=4.8\right.$ vs. $\left.F_{0.05}=4.0\right)$, but not in females $\left(\mathrm{F}=1.2\right.$ vs. $\left.\mathrm{F}_{0.05}=4.2\right)$. The estimated location of the male 6-week body mass QTL is very close to the estimated locations of the tail length QTL (Table 3).

Pooling the sexes, the single-QTL analysis of 10week tail length was highly significant $(F=75.3$ vs. $\left.\mathrm{F}_{0.01}=5.4\right)$ and yielded similar estimates of location and effect (location: $55 \mathrm{cM}$; 95\% confidence interval: $46 \mathrm{cM}$ to $56 \mathrm{cM}$; additive effect: $-0.65 \pm 0.05$; dominance effect: $0.15 \pm 0.08$ ) to the sex-specific analyses and to the original $F_{2}$ population. In the two-QTL analysis of the data from both sexes combined, the second QTL was not significant $\left(\mathrm{F}=2.9\right.$ vs. $\mathrm{F}_{0.05}=$ 4.0); similarly, composite interval mapping revealed a single significant peak (data not shown). It is possible that the second tail-length QTL identified in 
Table 4. Effect of genotype on skeletal dimensions in a sample of 30 individuals from the new $F_{2}$ population that were not recombinant between D1Mit30 and D1Mit57. Additive and dominance effects are as defined in Table 3; n.s. = not significant at $\alpha=0.05$

\begin{tabular}{|c|c|c|c|c|c|}
\hline & \multicolumn{2}{|c|}{ Additive effect $\left(\sigma_{p}\right)$} & \multicolumn{2}{|c|}{ Dominance effect $\left(\sigma_{p}\right)$} & \multirow{2}{*}{$\begin{array}{l}\text { P.E. }^{a} \\
(\%)\end{array}$} \\
\hline & Estimate & $P$-value & Estimate & $P$-value & \\
\hline \multicolumn{6}{|c|}{ Traits associated with axial length } \\
\hline Tail length & $-0.74 \pm 0.10$ & $<0.0001$ & $0.35 \pm 0.16$ & 0.0464 & 6.8 \\
\hline First vertebra length ${ }^{\mathrm{b}}$ & $-0.22 \pm 0.11$ & n.s. & $-0.33 \pm 0.22$ & n.s. & 2.9 \\
\hline First vertebra width ${ }^{b}$ & $-0.01 \pm 0.22$ & n.s. & $-0.01 \pm 0.37$ & n.s. & 0.0 \\
\hline Tail (caudal) bone length ${ }^{\mathrm{b}}$ & $-0.39 \pm 0.12$ & 0.014 & $-0.02 \pm 0.24$ & n.s. & 4.0 \\
\hline \multicolumn{6}{|l|}{ Long bones } \\
\hline Humerus length & $-0.48 \pm 0.10$ & 0.0004 & $0.19 \pm 0.19$ & n.s. & 4.2 \\
\hline Ulna length & $-0.27 \pm 0.14$ & 0.054 & $-0.10 \pm 0.21$ & n.s. & 2.1 \\
\hline Femur length & $-0.37 \pm 0.15$ & 0.018 & $0.19 \pm 0.23$ & n.s. & 3.1 \\
\hline Tibia length & $-0.43 \pm 0.08$ & $<0.0001$ & $0.04 \pm 0.14$ & n.s. & 3.2 \\
\hline \multicolumn{6}{|l|}{ Flat bones } \\
\hline Skull length & $0.05 \pm 0.19$ & n.s. & $0.12 \pm 0.32$ & n.s. & -0.2 \\
\hline Skull width & $-0.12 \pm 0.17$ & n.s. & $-0.02 \pm 0.28$ & n.s. & 1.3 \\
\hline Mandible length & $-0.48 \pm 0.14$ & 0.0045 & $0.06 \pm 0.24$ & n.s. & 3.2 \\
\hline Scapula length & $-0.30 \pm 0.11$ & 0.0127 & $-0.05 \pm 0.17$ & n.s. & 2.5 \\
\hline Pelvic girdle length & $-0.41 \pm 0.15$ & 0.0169 & $0.22 \pm 0.25$ & n.s. & 3.1 \\
\hline
\end{tabular}

a Proportionate effect $=$ the difference between the means of the two homozygotes divided by the mean of the DBA/2J homozygote.

b Sample size for first vertebra length and width is 28 , while that for the tail bone is 24 owing to damaged or lost samples.

the original $\mathrm{F}_{2}$ population was not within the introgressed region. When the data for both sexes were combined, there was a significant QTL affecting 10week body mass $\left(\mathrm{F}=5.3\right.$ vs. $\left.\mathrm{F}_{0.01}=5.3\right)$ at the same location as the tail length QTL (i.e., $55 \mathrm{cM}$ ) with estimated effects similar to those observed for the mass QTL in the original $\mathrm{F}_{2}$ population (additive effect: $-0.14 \pm 0.05$; dominance effect: $0.06 \pm 0.07$ )

Effects of QTL on skeletal dimensions. In the sample of mice in which skeletal dimensions were measured, the additive effect of the QTL on tail length was similar to that observed in the new $F_{2}$ population, although C57 allele was partially dominant as in the females from the original population (Table 4). The QTL did not have significant effects on the dimensions of the first vertebra, but did have a significant effect on the length of the $10^{\text {th }}$ caudal bone; the magnitude of this effect was less than that observed for tail length (Table 4). While the QTL had an effect on the length of the tail bone, no effect on the number of bones in the tail was detected (DBA/ DBA: $31.5 \pm 0.6 ; \quad$ DBA/C57: $32.5 \pm 0.6 ; \quad$ C57/C57: $\left.32.7 \pm 0.6 ; \chi_{2}{ }^{2}=1.16, P=0.56\right)$. It is not clear to what extent the variation in the number of bones counted (range: 29-34) was due to measurement error (i.e., the loss of extremely small bones).

There was a significant effect of genotype on the lengths of the humerus, femur, tibia, mandible, scapula, and pelvic girdle, but not on the ulna or on the dimensions of the skull (Table 4). Where there was a significant effect of genotype on a skeletal dimension, the additive effect was in the same di- rection as for tail length but smaller in magnitude. Estimates of the dominance effect varied greatly and were not significant for any skeletal measurement other than tail length.

\section{Discussion}

The present study confirmed the previous finding of a QTL affecting tail length on Chr 1 (Morris et al. 1999) by additional genotyping of samples from the original study, as well as the production of a partially congenic strain and a new $\mathrm{F}_{2}$ population. Estimates of the additive effects of the QTL were similar to that obtained in the original study (additive effect $=0.65$ standard deviation units; Morris et al. 1999). However, the region of strong support for the QTL was approximately halved, reducing from the region between D1Mit7 and D1Mit57 (Morris et al. 1999) to an approximately 8-cM region between about D1Mit30 and D1Mit57; this region was consistently supported by both bootstrapping and composite interval mapping in both $\mathrm{F}_{2}$ populations and in both sexes. The estimated additive effect on tail length is similar in both sexes and increases with age, suggesting that this QTL predominantly influences the middle and late growth periods (sensu Vaughn et al. 1999), although the significant effect on 3-week tail length in males indicates that it also affects early growth.

Examination of the effects of the QTL on skeletal traits suggested that the effect on tail length was due to an increase in bone length rather than bone number. Furthermore, the QTL was found to influ- 
ence the length of a variety of bones including both long and flat bones. The estimated effects on individual bones were in the same direction as that on tail length but were generally smaller, perhaps because tail length represents the cumulative effect on a number of bones. Because individuals with recombination within the QTL region were mated for progeny testing, it was not possible to refine the position of the QTL or test for multiple QTL affecting the skeletal traits. As a result, the possibility that the effects on various bones are due to separate QTL from that or those affecting tail length cannot be ruled out. However, the offspring of the recombinant $F_{2}$ individuals will provide a powerful resource to address this issue.

Comparisons with other studies. The direction of QTL effects observed in this study is as expected, given that the femurs of C57 females are 7\% longer than those of DBA/2J females at 12 months of age (Beamer et al. 1996; see also Drake et al. 2001). Drake et al. (2001) conducted a genome-wide search for QTL affecting femoral bone dimensions in an $\mathrm{F}_{2}$ population derived from these two inbred strains (i.e., the same as the parental strains used in this study), but did not detect any QTL on Chr 1. These authors did find two QTL affecting femur length on other chromosomes, and one of these shows the same direction of effect as observed in this study (i.e., C57 > DBA). The magnitude of the effect of both QTL (a difference of about 3\% between homozygotes; Drake et al. 2001) is similar to that observed for femur length in this study, and thus it is not clear why Drake et al. (2001) did not detect the Chr 1 QTL; Drake et al. (2001) examined only females, and it is possible that the effect is less pronounced in this sex.

In an intercross between two inbred strains selected for high and low body size, Cheverud et al. (2001) identified seven QTL affecting tail length, one of which was located on Chr 1. The estimated location of this QTL is proximal to that of the QTL described in this study, and the confidence intervals from the two studies only just overlap. The Chr 1 QTL described by Cheverud et al. (2001) affects tail length in both sexes, as in this study, but does not have an effect on body mass at 10 weeks of age, consistent with the relatively weak or non-significant effects on body mass observed in this study. Klingenberg et al. (2001) and Corva et al. (2001) performed genome-wide scans for QTL affecting mandible size and femur length, respectively (among other traits), but did not detect QTL for these traits on Chr 1.
Candidate genes. Within the region of the QTL described in this study, there are at least two genes for which targeted mutation results in skeletal abnormalities: myogenin (Myog) and the paired-related homeobox 1 gene (Prx1) (Hasty et al. 1993; Martin et al. 1995). However, in both cases the phenotypic patterns are substantially different from those observed in this study. A number of candidate genes that might influence the growth of bones are either proximal (Gli2, Igfbp2, Igfbp5, Ihh, Inha, Inhbb, Nppc, Pax3, and Tnfrsf11a) or distal (TGF 32 ) to the QTL described here (Ensembl 2002; MGD 2002). A number of other candidate genes for bone growth (discussed by Ducy and Karsenty 1998; Rosen et al. 2000; Drake et al. 2001; Wagner and Karsenty 2001) are not located on Chr 1 (Ensembl 2002; MGD 2002). The absence of strong candidate genes within the region of the QTL highlights the possibility that its effects may be due to as-yet-unknown gene(s).

\section{Acknowledgments}

We are grateful to Katie McDonald for technical advice and to two anonymous referees for helpful comments. We thank the Biotechnology and Biological Research Council, the Medical Research Council, the Royal Society, and the Natural Sciences and Engineering Research Council of Canada for financial support.

\section{References}

1. Barsh GS, Farooqi IS, O'Rahilly S (2000) Genetics of body-weight regulation. Nature 404, 644-651

2. Beamer WG, Donahue LR, Rosen CJ, Baylink DJ (1996) Genetic variability in adult bone density among inbred strains of mice. Bone 18, 397-403

3. Bünger L, Laidlaw A, Bulfield G, Eisen EJ, Medrano JF et al. (2001) Inbred lines of mice derived from longterm growth selected lines: unique resources for mapping growth genes. Mamm Genome 12, 678-686

4. Cheverud JM, Vaughn TT, Pletscher LS, Peripato AC, Adams ES et al. (2001) Genetic architecture of adiposity in the cross of LG/J and SM/J inbred mice. Mamm Genome 12, 3-12

5. Churchill GA, Doerge RW (1994) Empirical threshold values for quantitative trait mapping. Genetics 138, 963-971

6. Corva PM, Medrano JF (2001) Quantitative trait loci (QTLs) mapping for growth traits in the mouse: a review. Genet Sel Evo1 33, 105-132

7. Corva PM, Horvat S, Medrano JF (2001) Quantitative trait loci affecting growth in high growth (hg) mice. Mamm Genome 12, 284-290

8. Darvasi A (1997) The effect of selective genotyping on QTL mapping accuracy. Mamm Genome 8, 67-68 
9. Drake TA, Hannani K, Kabo JM, Villa V, Krass K et al. (2001) Genetic loci influencing natural variations in femoral bone morphometry in mice. J Orthop Res 19, 511-517

10. Ducy P, Karsenty G (1998) Genetic control of cell differentiation in the skeleton. Curr Opin Cell Biol 10, 614-619

11. Ensembl (2002) Ensembl Mouse Genome Server Database. World Wide Web (URL: http://www.ensembl.org/ Mus_musculus//, May 2002

12. Green P, Falls K, Crooks S (1990) Documentation for CRI-MAP, version 2.4. World Wide Web (URL:http:// compgen.rutgers.edu/multimap/crimap//

13. Hasty P, Bradley A, Morris JH, Edmondson DG, Venuti JM et al. (1993) Muscle deficiency and neonatal death in mice with a targeted mutation in the myogenin gene. Nature 364, 501-506

14. Klingenberg CP, Leamy LJ, Routman EJ, Cheverud JM (2001) Genetic architecture of mandible shape in mice: effects of quantitative trait loci analyzed by geometric morphometrics. Genetics 157, 785-802

15. Mackay TFC (2001) The genetic architecture of quantitative traits. Annu Rev Genet 35, 303-339

16. Martin JF, Bradley A, Olson EN (1995) The paired-like homeobox gene Mhox is required for early events of skeletogenesis in multiple lineages. Genes Dev 9, $1237-1249$

17. MGD (2002) Mouse Genome Informatics Web Site, The Jackson Laboratory, Bar Harbor, Maine. World
Wide Web (URL: http://www.informatics.jax.org/), May 2002

18. Morris KH, Ishikawa A, Keightley PD (1999) Quantitative trait loci for growth traits in C57BL/6J $\times$ DBA/2J mice. Mamm Genome 10, 225-228

19. Rosen CJ, Churchill GA, Donahue LR, Shultz KL, Burgess JK et al. (2000) Mapping quantitative trait loci for serum insulin-like growth factor-1 levels in mice. Bone 27, 521-528

20. SAS Institute (1989) SAS/STAT User's Guide, Version 6, 4th edn, Vol. 2. (Cary, NC: SAS Institute Inc.)

21. Seaton G, Haley C, Knott S, Kearsey M, Visscher P (2002) QTL express. World Wide Web (URL: http:// qtl.cap.ed.ac.uk), May 2002

22. Sims H, Baron R (2000) Bone cells and their function. In Skeletal Growth Factors, E. Canalis, ed. (Philadelphia: Lippincott, Williams and. Wilkins), pp 1-16

23. Vaughn TT, Pletscher LS, Peripato A, King-Ellison K, Adams E et al. (1999) Mapping quantitative trait loci for murine growth: a closer look at genetic architecture. Genet Res 74, 313-322

24. Visscher PM, Thompson R, Haley CS (1996) Confidence intervals in QTL mapping by bootstrapping. Genetics 143, 1013-1020

25. Wagner EF, Karsenty G (2001) Genetic control of skeletal development. Curr Opin Genet Dev 11, 527-532

26. Wang S, Basten CJ, Zeng Z-B (2001) Windows QTL Cartographer v. 1.21. World Wide Web (URL:http:// statgen.ncsu.edu/qtlcart/cartographer.html) 